Forum 2016 · 31:258

DOI 10.1007/s12312-016-0070-4

C) Springer-Verlag Berlin Heidelberg 2016

Schleswig-Holsteinische

Krebsgesellschaft e.V.

Schleswig Holsteinische Krebsgesellschaft e.V.

\title{
Kommunikationstraining für von Krebs betroffene Paare
}

\author{
Schleswig-Holsteinische Krebsgesellschaft zieht \\ positive Bilanz
}

Februar erstmals ein Kommunikationstraining für Paare angeboten, von denen mindestens ein Partner von einer Krebserkrankung betroffen ist. Unter der Leitung von Dipl.-Psych. Karin Lausmann und Dipl.-Psych. Lars Mandelkow gingen die teilnehmenden Paare folgenden Fragen nach: Wie erleben wir unsere Partnerschaft seit der Diagnose Krebs? Was hat sich verändert? Wie reden wir miteinander über die Krankheit, unsere Ängste und Hoffnungen? Was können wir als Paar zusammen dafür tun, dass wir die Zeit der Krankheit gut überstehen?

\section{》IIm Mittelpunkt der Übungen stand das Verständnis für den Partner}

Das Training, an dem insgesamt vier Paare teilnahmen, bestand aus Vorträgen, Paarübungen und dem Austausch untereinander. Im Mittelpunkt der Übungen stand das Verständnis für den Partner ohne Verzicht auf eigene Bedürfnisse sowie die Autonomie beider Partner innerhalb der Beziehung. Ziel des Trainings war eine Stärkung der gegenseitigen Unterstützung und die Reduktion von Belastungen. Die Paarübungen fanden in unterschiedlichen Räumen statt, sodass die Paare ihre Themen im vertrauten Rahmen besprechen konnten.

$\mathrm{Zu}$ Beginn lernten die Teilnehmer die grundlegenden Regeln der Gesprächsführung kennen, um anschließend das Gelernte zu üben. „Anfangs war durchaus auch Skepsis zu spüren, da einige Teilnehmer die vorgestellten Regeln bereits kannten“, berichtet Karin Lausmann. Als es in einer anschließenden Paarübung dann aber darum ging, diese Regeln auch anzuwenden, kamen die Schwierigkeiten zutage und großes Erstaunen stellte sich ein. Am Ende des Tages konnten alle Paare neue Erkenntnisse und Anstöße mit in ihre Beziehung nehmen.

\section{Kontakt}

Schleswig Holsteinische

Krebsgesellschaft e.V.

Sonja Kulschewski

Alter Markt 1-2

24103 Kiel

Tel.: 0431/800 1082

kulschewski@krebsgesellschaft-sh.de

www.krebsgesellschaft-sh.de 